\title{
Diophantine quadruples with values in $k$-generalized Fibonacci numbers
}

\author{
Carlos Alexis Gómez Ruiz ${ }^{*}$ and Florian Luca ${ }^{\dagger}$
}

February 20, 2017

\begin{abstract}
We consider for integers $k \geq 2$ the $k$-generalized Fibonacci sequences $F^{(k)}:=\left(F_{n}^{(k)}\right)_{n \geq 2-k}$, whose first $k$ terms are $0, \ldots, 0,1$ and each term afterwards is the sum of the preceding $k$ terms. In this paper, we show that there does not exist a quadruple of positive integers $a_{1}<a_{2}<a_{3}<a_{4}$ such that $a_{i} a_{j}+1(i \neq j)$ are all members of $F^{(k)}$.
\end{abstract}

Key words and phrases. Generalized Fibonacci numbers, Diophantine quadruples.

2010 Mathematics Subject Classification. 11B39, 11D61.

\section{Introduction}

A Diophantine $m$-tuple is a set $\left\{a_{1}, \ldots, a_{m}\right\}$ of $m$ positive rational numbers or integers, with the property that the product of any two of its distinct elements plus one is a square; i.e., such that $a_{i} a_{j}+1$ is a square for all $1 \leq i<j \leq m$. Diophantus presented the first known rational quadruple

$$
\left\{\frac{1}{16}, \frac{33}{16}, \frac{17}{4}, \frac{105}{16}\right\}
$$

and long after Fermat found the integer quadruple $\{1,3,8,120\}$. There are infinitely many Diophantine quadruples of integers, one such parametric family being known to Euler:

$$
\{a, b, a+b+2 t, 4 t(t+a)(t+b)\}, \text { where } a b+1=t^{2} .
$$

*Departamento de Matemáticas, Universidad del Valle, 25360 Cali, Calle 13 No 100-00, Colombia. e-mail: carlos.a.gomez@correounivalle.edu.co

†School of Mathematics, University of the Witwatersrand, Private Bag X3, Wits 2050, South Africa; Max Planck Institute for Mathematics Vivatgasse 7, 53111 Bonn, Germany, Department of Mathematics Faculty of Sciences University of Ostrava 30. Dubna 22, 701 03 Ostrava 1, Czech Republic, e-mail: florian.luca@wits.ac.za 
On the other hand, Arkin, Hoggatt and Strauss [1] observed that any Diophantine triple can be extended to a Diophantine quadruple. More precisely, if $\{a, b, c\}$ is a Diophantine triple with $a b+1=t^{2}, a c+1=u^{2}, \quad b c+1=v^{2}$, where $t, u, v$ are positive integers, then setting $d:=a+b+c+2 a b c+2 t u v$, the set $\{a, b, c, d\}$ is a Diophantine quadruple. Regarding Diophantine $m$-tuples with $m \geq 5$, Dujella [8], proved that there is no Diophantine sextuple and that there can be at most finitely many Diophantine quintuples. In [9], he showed that $10^{1930}$ is an upper bound on the number of Diophantine quintuples. This bound has been recently reduced to $5.441 \times 10^{26}$ by $\mathrm{Cipu}$ and Trudgian in [6].

A natural generalization of the problem described above is to replace the squares by the members of some interesting sequence of integers. So, let $\mathbf{U}:=\left(U_{n}\right)_{n \geq 0}$ be a sequence of integers. We say that a finite set $\left\{a_{1}, \ldots, a_{m}\right\}$ of positive integers is a Diophantine $m$-tuple with values in $\mathbf{U}$ if $a_{i} a_{j}+1$ is a member of $\mathbf{U}$ for all $1 \leq i<j \leq m$. We assume that $m \geq 3$ to avoid trivialities. Diophantine $m$-tuples associated to the sequences of higher (than 2) powers of integers of fixed or variable exponents were studied in $[4,5,16,17,19]$, while Diophantine $m$-tuples with members in nondegenerate binary recurrences were studied by Fuchs, Luca and Szalay in [13]. Later, Luca and Szalay showed that there are no Diophantine triples with values in the Fibonacci sequence (see [20]) and that the only Diophantine triple with values in the Lucas companion $\left(L_{n}\right)_{n \geq 0}$ of the Fibonacci sequence is $(a, b, c)=(1,2,3)$ (see [21]). Very little is known about Diophantine $m^{-}$ tuples with values in linear recurrences of order greater than two. The current authors worked with the Tribonacci sequence $\left(T_{n}\right)_{n \geq 0}$ proving in [15] the following theorem.

Theorem 1. There do not exist positive integers $a_{1}<a_{2}<a_{3}<a_{4}$ such that $a_{i} a_{j}+1=T_{n_{i, j}}$, with $1 \leq i<j \leq 4$, for some integers positive $n_{i, j}$.

The above result was complimented by Fuchs, Hutle, Irmak, Luca and Szalay [12], who showed that there are at most finitely many Diophantine triples with values in the Tribonacci sequence. At the referee's suggestion, we did a computational search with Mathematica which showed that in fact there are no Diophantine triples $\left\{a_{1}, a_{2}, a_{3}\right\}$ with values in $k$-generalized Fibonacci numbers in the range

$$
3 \leq k \leq 20, \quad 1 \leq a_{1} \leq 2000, \quad a_{2} \leq 10^{5} \quad \text { and } \quad a_{3} \leq 10^{6} .
$$

We propose the following conjecture.

Conjeture 1. There are no Diophantine triples with values in $F^{(k)}$ for any integer $k \geq 2$.

In this paper, we extend the conclusion of Theorem 1 from Tribonacci numbers to $k$-generalized Fibonacci sequences $F^{(k)}$ for any $k \geq 3$.

Our main result is the following theorem. 
Theorem 2. Let $k \geq 2$ be a fixed integer. There do not exist positive integers $a_{1}<a_{2}<a_{3}<a_{4}$ such that

$$
a_{i} a_{j}+1 \in F^{(k)} \quad \text { for all } \quad 1 \leq i<j \leq 4
$$

\section{Preliminary results on $k$-Fibonacci num- bers}

For an integer $k \geq 2$, the $k$-generalized Fibonacci sequence $F^{(k)}:=\left(F_{n}^{(k)}\right)_{n \geq 2-k}$, satisfies the $k$-th order linear recurrence

$$
F_{n+k}^{(k)}=F_{n+k-1}^{(k)}+\cdots+F_{n}^{(k)} \quad(n \geq 2-k),
$$

with $F_{2-k}^{(k)}=F_{1-k}^{(k)}=\cdots=F_{0}^{(k)}=0$ and $F_{1}^{(k)}=1$. We note that some authors work with a shift of the above sequence, namely the one for which $F_{i}^{(k)}=0$ for $0 \leq i \leq k-2$ and $F_{k-1}^{(k)}=1$. We prefer to work with our version for which the first nonzero value is $F_{1}^{(k)}=1$.

We shall refer in general to $F_{n}^{(k)}$ as the $n$th $k$-Fibonacci number. For $k=2$, we have $F_{n}^{(2)}=F_{n}$, the familiar $n$th Fibonacci number. For $k=3$ such numbers are called Tribonacci numbers. They are followed by the Tetranacci numbers for $k=4$, and so on.

The first direct observation is that the first $k+1$ non-zero terms in $F^{(k)}$ are powers of two, namely

$$
F_{1}^{(k)}=1 \quad \text { and } \quad F_{n}^{(k)}=2^{n-2} \quad \text { for all } \quad 2 \leq n \leq k+1,
$$

while the next term is $F_{k+2}^{(k)}=2^{k}-1$. In fact, $F_{n}^{(k)}<2^{n-2}$ for all $n \geq k+2$ (see [2]). Cooper and Howard given the following nice formula for $F_{n}^{(k)}$ valid for all $n \geq k+2$ (see [7]):

Lemma 1. For $k \geq 2$ and $n \geq k+2$,

$$
F_{n}^{(k)}=2^{n-2}+\sum_{j=1}^{\left\lfloor\frac{n+k}{k+1}\right\rfloor-1} C_{n, j} 2^{n-(k+1) j-2},
$$

where

$$
C_{n, j}=(-1)^{j}\left[\left(\begin{array}{c}
n-j k \\
j
\end{array}\right)-\left(\begin{array}{c}
n-j k-2 \\
j-2
\end{array}\right)\right] .
$$

Here, we used the convention that $\left(\begin{array}{l}a \\ b\end{array}\right)=0$ if either $a<b$ or if one of $a$ or $b$ is negative and denote $\lfloor x\rfloor$ the greatest integer less than or equal to $x$. 


\section{$2.1 \quad$ Known properties of $F^{(k)}$}

We recall some known results concerning $F^{(k)}$. Clearly, $F^{(k)}$ is a linearly recurrent sequence of characteristic polynomial

$$
\Psi_{k}(X)=X^{k}-X^{k-1}-\cdots-X-1 .
$$

Note that by putting

$$
\psi_{k}(X)=(X-1) \Psi_{k}(X)=X^{k+1}-2 X^{k}+1,
$$

we get a new polynomial which has the same roots that $\Psi_{k}(X)$ together with an additional root at $X=1$.

The polynomial $\Psi_{k}(X)$ has only one positive real zero $\alpha:=\alpha(k)$ which is located in the interval $[1,2]$. In fact, in Lemma 2.3 in [18], it was shown

$$
2\left(1-2^{-k}\right)<\alpha(k)<2, \quad \text { for all } \quad k \geq 2,
$$

a fact rediscovered by Wolfram [24]. In particular, $(\alpha(k))_{k \geq 2}$ converges to 2 as $k$ tends to infinity. Miles [22] and Miller [23] showed that $\Psi_{k}(X)$ has only simple roots and all roots different from $\alpha(k)$ are inside the unit circle. In particular, $\Psi_{k}(X)$ is an irreducible polynomial over $\mathbb{Q}[X]$.

To simplify notation, we omit the dependence on $k$ of $\alpha$. We consider for $k \geq 2$, the function $f_{k}(z):=(z-1) /(2+(k+1)(z-2))$. With this notation, Dresden and Du proved in [10] that

$$
F_{n}^{(k)}=\sum_{i=1}^{k} f_{k}\left(\alpha_{i}\right) \alpha_{i}^{n-1} \quad \text { and } \quad\left|F_{n}^{(k)}-f_{k}(\alpha) \alpha^{n-1}\right|<\frac{1}{2},
$$

where $\alpha=: \alpha_{1}, \alpha_{2}, \ldots, \alpha_{k}$ are all the zeros of $\Psi_{k}(X)$. The expression on the left-hand side is known as the Binet formula for $F_{n}^{(k)}$. Furthermore, the inequality on the right-hand side in (5) shows that the contribution of the zeros of $\Psi_{k}(X)$ which are inside the unit circle to $F_{n}^{(k)}$ is very small. Also, it is easy to prove that the numbers $f_{k}(\alpha)$ and $f_{k}\left(\alpha_{i}\right)$ for $i=2, \ldots, k$ satisfy the inequalities

$$
\left.1 / 2 \leq f_{k}(\alpha) \leq 3 / 4 \quad \text { and } \quad \mid f_{k}\left(\alpha_{i}\right)\right) \mid<1, \quad \text { for } \quad i=2, \ldots, k,
$$

for all $k \geq 2$.

Finally, it was proved in [3] that

$$
\alpha^{n-2} \leq F_{n}^{(k)} \leq \alpha^{n-1} \quad \text { holds for all } n \geq 1 .
$$

\section{The proof of Theorem 2}

Let $a_{1}<a_{2}<a_{3}<a_{4}$ be a Diophantine quadruple associated to the $k^{-}$ generalized Fibonacci sequence $F^{(k)}$. Here, we assume that $k \geq 2$. Then

$$
a_{1} a_{2}+1=F_{x}^{(k)}, \quad a_{2} a_{3}+1=F_{y}^{(k)}, \quad a_{3} a_{4}+1=F_{z}^{(k)}, \quad a_{1} a_{4}+1=F_{w}^{(k)},
$$


hold for some integers positive $x, y, z$ and $w$. Combining the above equalities (8), we obtain that

$$
\left(F_{x}^{(k)}-1\right)\left(F_{z}^{(k)}-1\right)=\left(F_{y}^{(k)}-1\right)\left(F_{w}^{(k)}-1\right),
$$

where is easy see that

$$
4 \leq x<\min \{y, w\} \leq \max \{y, w\}<z .
$$

From inequalities (7), we deduce that

$$
\alpha^{n-2.5}<F_{n}^{(k)}-1<\alpha^{n-1}, \quad \text { for all } \quad n \geq 4 .
$$

Hence, it is plain that

$$
\begin{aligned}
\alpha^{x+z-5} & <\left(F_{x}^{(k)}-1\right)\left(F_{z}^{(k)}-1\right)<\alpha^{x+z-2} \\
\alpha^{y+w-5} & <\left(F_{y}^{(k)}-1\right)\left(F_{w}^{(k)}-1\right)<\alpha^{y+w-2} .
\end{aligned}
$$

Considering the above two inequalities and equality (9), we get

$$
|(x+z)-(y+w)| \leq 2 .
$$

We analyze the Diophantine equation (9), subjected to the conditions given in (10) and (11). We distinguish two cases, namely:

$$
|(x+z)-(y+w)|=1 \text { or } 2 \quad \text { and } \quad x+z=y+w .
$$

\subsection{The case $|(x+z)-(y+w)|=1$ or 2}

We use formula (5) to write

$$
F_{n}^{(k)}=f_{k}(\alpha) \alpha^{n-1}+e_{k}(n), \quad \text { where } \quad\left|e_{k}(n)\right|<1 / 2 .
$$

Using (12), we can rewrite equation (9) as

$$
\begin{aligned}
f_{k}(\alpha)^{2} \alpha^{x+z-2}-f_{k}(\alpha)^{2} \alpha^{y+w-2} & =f_{k}(\alpha)\left(1-e_{k}(z)\right) \alpha^{x-1}+f_{k}(\alpha)\left(1-e_{k}(x)\right) \alpha^{z-1} \\
& +f_{k}(\alpha)\left(e_{k}(w)-1\right) \alpha^{y-1}+f_{k}(\alpha)\left(e_{k}(y)-1\right) \alpha^{w-1} \\
& +e_{k}(x)+e_{k}(z)-e_{k}(y)-e_{k}(w) \\
& -e_{k}(x) e_{k}(z)+e_{k}(y) e_{k}(w) .
\end{aligned}
$$

Dividing both sides of above equation by $f_{k}(\alpha)^{2} \alpha^{x+z-2}$ and taking absolute values, we get

$$
\begin{aligned}
\left|1-\alpha^{-(x+z-y-w)}\right| & <\frac{1.5}{f_{k}(\alpha)}\left(\frac{1}{\alpha^{z-1}}+\frac{1}{\alpha^{x-1}}+\frac{\alpha^{y-z}}{\alpha^{x-1}}+\frac{\alpha^{w-z}}{\alpha^{x-1}}\right)+\frac{2.5 f_{k}(\alpha)^{-2}}{\alpha^{x+z-2}} \\
& <\frac{1}{\alpha^{x-1}}\left(\frac{3}{f_{k}(\alpha)}\left(1+\frac{1}{\alpha}\right)+\frac{5}{2 f_{k}(\alpha)^{2} \alpha^{6}}\right) \\
& <\frac{10}{\alpha^{x-1}}
\end{aligned}
$$


where we have used (10), and the facts that $\left|e_{k}(n)-1\right|<3 / 2$ and $f_{k}(\alpha)>1 / 2$. By inequality (11) and the fact that $x+z \neq y+w$, we obtain

$$
\min _{|x+z-y-w| \leq 2}\left|1-\alpha^{-(x+z-y-w)}\right|=1-\alpha^{-1}>0.46 .
$$

Thus, by (10), (13) and (14), we get $x=4$.

Hence, equation (9) becomes

$$
3 F_{z}^{(k)}-F_{\lambda}^{(k)} F_{\delta}^{(k)}=4-F_{\lambda}^{(k)}-F_{\delta}^{(k)}, \quad \lambda:=\min \{y, w\} \leq \delta:=\max \{y, w\} .
$$

Replacing $F_{z}^{(k)}, F_{\lambda}^{(k)}, F_{\delta}^{(k)}$ according to the equation (12) in the above equation (15), we conclude that

$$
\begin{aligned}
3 f_{k}(\alpha) \alpha^{z-1}-f_{k}(\alpha)^{2} \alpha^{\lambda+\delta-2} & =f_{k}(\alpha)\left(e_{k}(\delta)-1\right) \alpha^{\lambda-1}+f_{k}(\alpha)\left(e_{k}(\lambda)-1\right) \alpha^{\delta-1} \\
& -e_{k}(\lambda)-e_{k}(\delta)+e_{k}(\lambda) e_{k}(\delta)-3\left(e_{k}(z)-1\right)+1 .
\end{aligned}
$$

Dividing both sides of above equation by $3 f_{k}(\alpha) \alpha^{z-1}$, and taking absolute values, we get

$$
\begin{aligned}
\left|1-3^{-1} f_{k}(\alpha) \alpha^{\lambda+\delta-z-1}\right| & <\frac{1 / 2}{\alpha^{z-\lambda}}+\frac{1 / 2}{\alpha^{z-\delta}}+\frac{27 /\left(12 f_{k}(\alpha) \alpha^{5}\right)}{\alpha^{z-5}} \\
& <\frac{1.4}{\alpha^{z-\delta}},
\end{aligned}
$$

where we used the fact that $z-5 \geq z-\lambda \geq z-\delta$ (by (10)). However, by inequality (11) and the fact that $x=4$, we obtain that $|\lambda+\delta-z-1| \leq 5$. We check that

$$
\min _{|\lambda+\delta-z-1| \leq 5}\left|1-3^{-1} f_{k}(\alpha) \alpha^{\lambda+\delta-z-1}\right|>0.09863 .
$$

Thus, combining (10), (16) and (17) we conclude that $z-\delta=1,2,3$ or 4 . Returning to inequality (11), we get that $5 \leq \lambda \leq 10$.

Going back to equality (9), we rewrite it as

$$
3 F_{z}^{(k)}-\left(F_{\lambda}^{(k)}-1\right) F_{\delta}^{(k)}=3-\left(F_{\lambda}^{(k)}-1\right) .
$$

Replacing $F_{z}^{(k)}, F_{\delta}^{(k)}$ according to (12) in (18), dividing by $\left(F_{\lambda}^{(k)}-1\right) f_{k}(\alpha) \alpha^{\delta-1}$ and taking value absolutes, we get

$$
\left|1-3\left(F_{\lambda}^{(k)}-1\right)^{-1} \alpha^{z-\delta}\right|<\frac{3}{\alpha^{\delta-1}} .
$$

By analyzing the minimum value of the left-hand side in (19), we get

$$
\min _{\substack{5 \leq \lambda \leq 10 \\ 1 \leq z-\delta \leq 4}}\left|1-3\left(F_{\lambda}^{(k)}-1\right)^{-1} \alpha^{z-\delta}\right|>0.127 .
$$

Hence, from inequalities (19) and (20) we conclude that $\delta \leq 6$ and, in particular, that $6 \leq z \leq 10$.

Let us record what we have proved so far. 
Lemma 2. Let $4 \leq x<\min \{y, w\} \leq \max \{y, w\}<z$ be positive integers such that $|x+z-y-w|=1,2$ and $\left(F_{x}^{(k)}-1\right)\left(F_{z}^{(k)}-1\right)=\left(F_{y}^{(k)}-1\right)\left(F_{w}^{(k)}-1\right)$, for all $k \geq 4$. Then

$$
x=4, \quad 5 \leq y, \quad w \leq 6 \quad \text { and } \quad 6 \leq z \leq 10 .
$$

To conclude this section, we show that there are no Diophantine quadruples associated to $F^{(k)}$, under the current assumptions. We first list the values of $F_{x}^{(k)}, F_{y}^{(k)}, F_{z}^{(k)}, F_{w}^{(k)}$, with $4 \leq k \leq 9$ and $x, y, z, w$ in the range given by Lemma 2 , which leads us to the conclusion that (9) has no solutions. So, there are no Diophantine quadruples with values in $F^{(k)}$, with $4 \leq k \leq 9$. Now, when $k \geq 10$, we note that $F_{t}^{(k)}=2^{t-2}$, for $t \in\{x, y, z, w\}$. But a quick verification in equation

$$
\left(2^{x-2}-1\right)\left(2^{z-2}-1\right)=\left(2^{y-2}-1\right)\left(2^{w-2}-1\right),
$$

with $x, y, z, w$ distinct integers in the ranges given by the previous lemma allows us to conclude that there are no Diophantine quadruples associated to $F^{(k)}$ in the case $k \geq 10$ either.

\subsection{The case $x+z=y+w$}

We first prove the following result:

Lemma 3. Inequality

$$
\left(F_{n+2}^{(k)}-1\right)\left(F_{n}^{(k)}-1\right) \leq\left(F_{n+1}^{(k)}-1\right)^{2}
$$

holds for all $n \geq 0$ and $k \geq 2$. Equality is obtained only for $n=0,1, k+1$.

Remark 1. The above result says (a little bit more than) that the sequence $F^{(k)}$ is "log-concave".

Proof. Let $k=2$. One checks that inequality (21) is an equality for $n=0,1,3$ and it is strict for $n=2$. Assume $n \geq 4$. Then inequality (21) is equivalent to

$$
F_{n+2} F_{n}-F_{n+1}^{2} \leq F_{n+2}+F_{n}-2 F_{n+1} .
$$

The right-hand side of (22) is

$$
\begin{aligned}
F_{n+2}+F_{n}-2 F_{n+1} & =\left(F_{n+1}+F_{n}\right)+F_{n}-2 F_{n+1} \\
& =2 F_{n}-F_{n+1} \\
& =2 F_{n}-\left(F_{n}+F_{n-1}\right) \\
& =F_{n}-F_{n-1} \\
& =F_{n-2},
\end{aligned}
$$

while the left-hand side of $(22)$ is $(-1)^{n+1}$. So, we get that inequality $(22)$ is equivalent to

$$
(-1)^{n+1} \leq F_{n-2}
$$


which holds with strict for all $n \geq 4$.

From now on we assume that $k \geq 3$. We note by Lemma 1 that

$$
F_{n}^{(k)}= \begin{cases}0 & \text { if } n=0, \\ 1 & \text { if } n=1, \\ 2^{n-2} & \text { if } 2 \leq n \leq k+1, \\ 2^{n-2}-(n-k) 2^{n-k-3} & \text { if } k+2 \leq n \leq 2 k+2\end{cases}
$$

We now start with the cases where $(21)$ is an equality. For $n=0,1$, both sides of inequality (21) are zero so Lemma 3 holds with equality. For $n=k+1$, we have

$$
F_{n}^{(k)}=F_{k+1}^{(k)}=2^{k-1}, \quad F_{n+1}^{(k)}=F_{k+2}^{(k)}=2^{k}-1, \quad F_{n+2}^{(k)}=F_{k+3}^{(k)}=2^{k+1}-3,
$$

so inequality (21) asserts that

$$
\left(2^{k+1}-4\right)\left(2^{k-1}-1\right) \leq\left(2^{k}-2\right)^{2},
$$

which is again an equality.

For $n=2, \ldots, k-1$, we have that

$$
\begin{aligned}
\left(F_{n+1}^{(k)}-1\right)^{2}-\left(F_{n}^{(k)}-1\right)\left(F_{n+2}^{(k)}-1\right) & =\left(2^{n-1}-1\right)^{2}-\left(2^{n-2}-1\right)\left(2^{n}-1\right) \\
& =\left(2^{2 n-2}-2^{n}+1\right)-\left(2^{2 n-2}-5 \cdot 2^{n-2}+1\right) \\
& =2^{n-2}>0,
\end{aligned}
$$

so inequality (21) is strict. For $n=k$, we have

$$
\begin{aligned}
\left(F_{n+1}^{(k)}-1\right)^{2}-\left(F_{n}^{(k)}-1\right)\left(F_{n+2}^{(k)}-1\right) & =\left(2^{k-1}-1\right)^{2}-\left(2^{k-2}-1\right)\left(2^{k}-2\right) \\
& =\left(2^{2 k-2}-2^{k}+1\right)-\left(2^{2 k-2}-6 \cdot 2^{k-2}+2\right) \\
& =2^{k-1}-1>0,
\end{aligned}
$$

so inequality (21) is strict.

For $n=k+2, \ldots, 2 k$, we have

$$
\begin{gathered}
F_{n}^{(k)}=2^{n-2}-(n-k) 2^{n-k-3}, \quad F_{n+1}^{(k)}=2^{n-1}-(n-k+1) 2^{n-k-2}, \\
F_{n+2}^{(k)}=2^{n}-(n-k+2) 2^{n-k-1},
\end{gathered}
$$

so

$$
\begin{aligned}
& \left(F_{n+1}^{(k)}-1\right)^{2}-\left(F_{n}^{(k)}-1\right)\left(F_{n+2}^{(k)}-1\right) \\
= & \left(2^{n-1}-(n-k+1) 2^{n-k-2}-1\right)^{2} \\
- & \left(2^{n-2}-(n-k) 2^{n-k-3}-1\right)\left(2^{n}-(n-k+2) 2^{n-k-1}-1\right) \\
= & 2^{2 n-2}-2^{n}\left((n-k+1) 2^{n-k-2}+1\right)+\left((n-k+1) 2^{n-k-2}+1\right)^{2} \\
& -2^{2 n-2}+2^{n-2}\left((n-k+2) 2^{n-k-1}+4(n-k) 2^{n-k-3}+5\right) \\
& -\left((n-k) 2^{n-k-3}+1\right)\left((n-k+2) 2^{n-k-1}+1\right) \\
= & 2^{n-2}+(n-k+1)^{2} 2^{2 n-2 k-4}+(n-k+1) 2^{n-k-1}+1 \\
& -(n-k)(n-k+2) 2^{2 n-2 k-4}-(5 n-5 k+8) 2^{n-k-3}-1 \\
= & 2^{n-2}-2^{2 n-2 k-4}-(n-k+4) 2^{n-k-3} \\
= & 2^{n-2}+2^{n-k-3}\left(2^{n-k-1}-((n-k-1)+5)\right) .
\end{aligned}
$$


Let $t:=n-k-1 \geq 1$. The inequality

$$
2^{t}-(t+5) \geq 0 \quad \text { holds for all } t \geq 3 \text {. }
$$

So, if $t \geq 3$, then $n-2=t+k-1 \geq k+2$, so the inequality

$$
\left(F_{n+1}^{(k)}-1\right)^{2}-\left(F_{n}^{(k)}-1\right)\left(F_{n+2}^{(k)}-1\right) \geq 2^{n-2} \geq 2^{k+2} \geq 32
$$

holds. If $t=2$, then $2^{t}-(t+5)=-3, n=k+3$, so we get that the inequality

$$
\left(F_{n+1}^{(k)}-1\right)^{2}-\left(F_{n}^{(k)}-1\right)\left(F_{n+2}^{(k)}-1\right)=2^{k+1}+2(-3)=2^{k+1}-6 \geq 10
$$

holds. Finally, if $t=1$, then $2^{t}-(t+5)=-4, n=k+2$, so we get that the inequality

$$
\left(F_{n+1}^{(k)}-1\right)^{2}-\left(F_{n}^{(k)}-1\right)\left(F_{n+2}^{(k)}-1\right)=2^{n-2}-4=2^{k}-4 \geq 4
$$

holds.

We record the weaker conclusion of what we have done so far namely that

$$
\left(F_{n+1}^{(k)}-1\right)^{2}-\left(F_{n}^{(k)}-1\right)\left(F_{n+2}^{(k)}-1\right) \geq 4
$$

holds for all $n=k+2, k+3, \ldots, 2 k$, which follows from (23), (24) and (25). We let $n=2 k+1$. Then

$$
\begin{aligned}
& F_{n}^{(k)}=F_{2 k+1}^{(k)}=2^{2 k-1}-(k+1) 2^{k-2}, \\
& F_{n+1}^{(k)}=F_{2 k+2}^{(k)}=2^{2 k}-(k+2) 2^{k-1}, \\
& F_{n+2}^{(k)}=F_{2 k+3}^{(k)}=2^{2 k+1}-(k+3) 2^{k}+1 .
\end{aligned}
$$

Computing we get

$$
\left(F_{n+1}^{(k)}-1\right)^{2}-\left(F_{n}^{(k)}-1\right)\left(F_{n+2}^{(k)}-1\right)=\left(2^{k-1}-1\right)^{2} \geq 9 .
$$

In particular, inequality (26) holds for $n=2 k+1$ as well.

Now we rewrite identity (12), according to (5), as

$$
F_{n}^{(k)}=c_{1} \alpha^{n}+e_{k}(n) \text {, with } e_{k}(n)=c_{2} \alpha_{2}^{n}+\cdots+c_{k} \alpha_{k}^{n}
$$

where $\alpha=: \alpha_{1}, \alpha_{2}, \ldots, \alpha_{k}$ are the zeros of characteristic polynomial $\Psi_{k}(x)$ and $c_{i}=f_{k}\left(\alpha_{i}\right) / \alpha_{i}$ for $i=1, \ldots, k$. We use the following facts:

(i) $\left|e_{k}(n)\right| \leq 1 / 2$ for all $n \geq 0$ (by inequality (5));

(ii) $F_{n+2}^{(k)}=2 F_{n+1}^{(k)}-F_{n+1-k}^{(k)}$ holds for all $n \geq k+1$ (which follows from (3)).

We write

$$
\begin{aligned}
& \left(F_{n+1}^{(k)}-1\right)^{2}-\left(F_{n}^{(k)}-1\right)\left(F_{n+2}^{(k)}-1\right) \\
= & \left(F_{n+1}^{(k)}\right)^{2}-F_{n}^{(k)} F_{n+2}^{(k)}+\left(F_{n+2}^{(k)}+F_{n}^{(k)}-2 F_{n+1}^{(k)}\right) \\
= & \left(c_{1} \alpha^{n+1}+e_{k}(n+1)\right)^{2}-\left(c_{1} \alpha^{n}+e_{k}(n)\right)\left(c_{1} \alpha^{n+2}+e_{k}(n+2)\right)+F_{n}^{(k)}-F_{n+1-k}^{(k)} \\
= & 2 c_{1} \alpha^{n+1} e_{k}(n+1)-c_{1} \alpha^{n} e_{k}(n+2)-c_{1} \alpha^{n+2} e_{k}(n)+c_{1}\left(1-\alpha^{1-k}\right) \alpha^{n} \\
+ & \left(e_{k}(n+1)^{2}-e_{k}(n) e_{k}(n+2)+e_{k}(n)-e_{k}(n+1-k)\right) \\
:= & W_{n}^{(k)}+\Delta_{n}^{(k)}
\end{aligned}
$$


where

$$
\begin{aligned}
W_{n}^{(k)} & :=2 c_{1} \alpha^{n+1} e_{k}(n+1)-c_{1} \alpha^{n} e_{k}(n+2)-c_{1} \alpha^{n+2} e_{k}(n)+c_{1}\left(1-\alpha^{1-k}\right) \alpha^{n} \\
\Delta_{n}^{(k)} & :=e_{k}(n+1)^{2}-e_{k}(n) e_{k}(n+2)+e_{k}(n)-e_{k}(n+1-k) .
\end{aligned}
$$

The important observation is that

$$
W_{n}^{(k)}=C_{1} \alpha^{n}+\sum_{i=2}^{k} C_{i}\left(\alpha \alpha_{i}\right)^{n}
$$

where

$$
C_{1}:=c_{1}\left(1-\alpha^{1-k}\right) \text { and } C_{i}:=-c_{1} c_{i}\left(\alpha-\alpha_{i}\right)^{2} ; \text { for } 2 \leq i \leq k .
$$

Therefore $\left(W_{n}^{(k)}\right)_{n \geq 0}$ is a linearly recurrent sequences of real numbers of order $k$ whose characteristic polynomial is

$$
\Psi_{W}(X)=(X-\alpha) \prod_{i=2}^{k}\left(X-\alpha \alpha_{i}\right) .
$$

Additionally $\left|\Delta_{n}^{(k)}\right| \leq 1.5$ (by (i)). So, since we want that

$$
\left(F_{n+1}^{(k)}-1\right)^{2}-\left(F_{n}^{(k)}-1\right)\left(F_{n+2}^{(k)}-1\right)>0,
$$

it follows, by calculation (27), that is suffices that the inequality

$$
W_{n}^{(k)}>1.5
$$

holds for all $n \geq k+2$. However, from (26), we know that the inequality

$$
W_{n}^{(k)}+\Delta_{n}^{(k)} \geq 4
$$

holds for $n=k+2, \ldots, 2 k+1$, therefore

$$
W_{n}^{(k)} \geq 2.5>1.5 \quad \text { holds for } \quad n=k+2, \ldots, 2 k+1 .
$$

It remains to write down explicitly the characteristic equation of $\left(W_{n}^{(k)}\right)_{n \geq 0}$. By equality (3)

$$
(X-1) \Psi_{k}(X)=X^{k+1}-2 X^{k}+1=(X-1)(X-\alpha) \cdots\left(X-\alpha_{k}\right),
$$

it follows, upon making the substitution $Y:=X / \alpha$, that

$$
Y^{k+1}-2 \alpha Y^{k}+\alpha^{k+1}=(Y-\alpha) \prod_{i=1}^{k}\left(Y-\alpha \alpha_{i}\right) .
$$

Hence,

$$
\Psi_{W}(Y)=\frac{Y^{k+1}-2 \alpha Y^{k}+\alpha^{k+1}}{Y-\alpha^{2}}=Y^{k}+r_{1} Y^{k-1}+\cdots+r_{k} .
$$


Thus,

$$
\left(Y-\alpha^{2}\right)\left(Y^{k}+r_{1} Y^{k-1}+\cdots+r_{k}\right)=Y^{k+1}-2 \alpha Y^{k}+\alpha^{k+1} .
$$

Identifying coefficients we get

$$
r_{1}-\alpha^{2}=-2 \alpha, \quad r_{2}-r_{1} \alpha^{2}=0, \quad r_{3}-r_{2} \alpha^{2}=0, \ldots, \quad-r_{k} \alpha^{2}=\alpha^{k+1} .
$$

Hence,

$$
r_{1}=\alpha^{2}-2 \alpha<0, \quad r_{2}=a_{1} \alpha^{2}<0, \quad r_{3}=a_{2} \alpha^{2}<0, \ldots, \quad r_{k}=-\alpha^{k-1}<-1 .
$$

Thus,

$$
W_{n+k}^{(k)}=\left(-r_{1}\right) W_{n+k-1}^{(k)}+\left(-r_{2}\right) W_{n+k-2}^{(k)}+\cdots+\left(-r_{k}\right) W_{n}^{(k)}
$$

holds for all $n \geq 0$, where all coefficients $-r_{1},-r_{2}, \ldots,-r_{k}$ are positive and the last one is larger than 1 . Well, but then, since $W_{n}^{(k)}>1.5$ for the values $n=k+2, \ldots, 2 k+1$, which are $k$ consecutive values for $n$, it follows, by recurrence (29), that

$$
W_{n+k}^{(k)} \geq\left(-r_{k}\right) W_{n} \geq W_{n}^{(k)} \quad \text { for all } \quad n \geq 0,
$$

so, by induction on $n$, we have that

$$
W_{n}^{(k)}>1.5 \quad \text { holds for all } \quad n \geq k+2 .
$$

As we have seen by calculation (27), this implies that (21) holds for all $n \geq k+2$ with strict, which finishes the proof of the lemma.

Let us now finish the proof of our theorem. We assume that there are integers $x, y, z, w, k$, as in inequalities (10) and $k \geq 2$, and

$$
\left(F_{x}^{(k)}-1\right)\left(F_{z}^{(k)}-1\right)=\left(F_{y}^{(k)}-1\right)\left(F_{w}^{(k)}-1\right), \quad \text { with } \quad x+z=y+w .
$$

Let $\lambda:=\min \{y, w\}$ and $\delta:=\max \{y, w\}$. Write $\lambda=x+h$. Then $z=\delta+h$, and we have

$$
\frac{F_{x+h}^{(k)}-1}{F_{x}^{(k)}-1}=\frac{F_{\lambda}^{(k)}-1}{F_{x}^{(k)}-1}=\frac{F_{z}^{(k)}-1}{F_{\delta}^{(k)}-1}=\frac{F_{\delta+h}^{(k)}-1}{F_{\delta}^{(k)}-1} .
$$

We thus get

$$
\begin{aligned}
\left(\frac{F_{x+1}^{(k)}-1}{F_{x}^{(k)}-1}\right)\left(\frac{F_{x+2}^{(k)}-1}{F_{x+1}^{(k)}-1}\right) \cdots\left(\frac{F_{x+h}^{(k)}-1}{F_{x+h-1}^{(k)}-1}\right) \\
=\left(\frac{F_{\delta+1}^{(k)}-1}{F_{\delta}^{(k)}-1}\right)\left(\frac{F_{\delta+2}^{(k)}-1}{F_{\delta+1}^{(k)}-1}\right) \cdots\left(\frac{F_{\delta+h}^{(k)}-1}{F_{\delta+h-1}^{(k)}-1}\right) .
\end{aligned}
$$


By our Lemma 3,

$$
\frac{F_{x+1}^{(k)}-1}{F_{x}^{(k)}-1} \geq \frac{F_{x+2}^{(k)}-1}{F_{x+1}^{(k)}-1} \geq \cdots \geq \frac{F_{\delta+1}^{(k)}-1}{F_{\delta}^{(k)}-1} .
$$

Further, the above string of inequalities implies

$$
\frac{F_{x+1}-1}{F_{x}-1}>\frac{F_{\delta+1}-1}{F_{\delta}-1} .
$$

Indeed, for if that would not be the case, then in (31) all intermediate inequalities are equalities. Assume first that $\delta \geq x+2$ and consider the first two equalities in the left-hand side in (31):

$$
\left(F_{x+2}^{(k)}-1\right)\left(F_{x}^{(k)}-1\right)=\left(F_{x+1}^{(k)}-1\right)^{2}, \quad\left(F_{x+3}^{(k)}-1\right)\left(F_{x+1}^{(k)}-1\right)=\left(F_{x+2}^{(k)}-1\right)^{2} .
$$

However, both equalities cannot be satisfied for the same value $x$ (by Lemma 3 , we must have $x=k+1$ for the first equality and $x=k$ for the second equality). So, (32) holds unless $\delta=x+1$. But this would mean that $w=x+1$, so

$$
\begin{aligned}
a_{1} a_{2}+1=F_{x}, & a_{1} a_{4}+1=F_{x+1}, \\
a_{2} a_{3}+1=F_{x+1}, & a_{3} a_{4}+1=F_{x+2},
\end{aligned}
$$

but then $F_{x}=a_{1} a_{2}+1<a_{1} a_{3}+1<a_{2} a_{3}+1=F_{x+1}$, showing that $a_{1} a_{3}+1$ cannot be in $F^{(k)}$. So, indeed we must have $\delta \geq x+2$ therefore (32) holds. A similar argument shows that

$$
\frac{F_{x+i}-1}{F_{x+i-1}-1}>\frac{F_{\delta+i}-1}{F_{\delta+i-1}-1} \quad \text { holds for } \quad i=1,2, \ldots, h
$$

and multiplying (33) for $i=1,2, \ldots, h$, we contradict (30) (in fact, we get that in (30), the left-hand side is larger than the right-hand side).

Thus, Theorem 2 is proved.

\section{Acknowledgement.}

We thank the referee for comments which improved the quality of this paper.

\section{References}

[1] J. Arkin, V. E. Hoggatt and E. G. Strauss, On Euler's solution of a problem of Diophantus, Fibonacci Quarterly 17 (1979), 333-339.

[2] J. J. Bravo and F. Luca, Powers of two in generalized Fibonacci sequences, Rev. Colombiana Mat. 46 (2012), 67-79.

[3] J. J. Bravo and F. Luca, On a conjecture about repdigits in $k$-generalized Fibonacci sequences, Publ. Math. Debrecen 82 (2013), 623-639. 
[4] Y. Bugeaud and A. Dujella, On a problem of Diophantus for higher powers, Math. Proc. Cambridge Philos. Soc. 135 (2003), 1-10.

[5] Y. Bugeaud and K. Gyarmati, On generalizations of a problem of Diophantus, Illinois J. Math. 48 (2004), 1105-1115.

[6] M. Cipu and T. Trudgian, Searching for Diophantine quintuples, Acta Arithmetica 173 (2016), 365-382.

[7] C. Cooper and F. T. Howard, Some identities for $r$-Fibonacci numbers, Fibonacci Quarterly 49 (2011), 231-243.

[8] A. Dujella, There are only finitely many Diophantine quintuples, J. reine angew. Math. 566 (2004), 183-214.

[9] A. Dujella, On the number of Diophantine m-tuples, Ramanujan J. 15 (2008), 37-46.

[10] G. P. Dresden and Zhaohui Du, A simplified Binet formula for $k$-generalized Fibonacci numbers, J. Integer Sequences 17 (2014), Article 14.4.7.

[11] G. Everest, A. van der Poorten, I. Shparlinski and T. Ward, Recurrence sequences, Mathematical Surveys and Monographs, 104 American Mathematical Society, Providence, RI, 2003.

[12] C. Fuchs, C. Hutle, N. Irmak, F. Luca and L. Szalay, Only finitely many Tribonacci Diophantine triples, Math. Slovaca, to appear.

[13] C. Fuchs, F. Luca and L. Szalay, Diophantine triples with values in binary recurrences, Ann. Sc. Norm. Super. Pisa Cl. Sc. (5), 7 (2008), 579-608.

[14] P. Gibbs, Some rational Diophantine sextuples, Glas. Mat. Ser. III 41(61) (2006), 195-203.

[15] C. A. Gómez Ruiz and F. Luca, Tribonacci Diophantine Quadruples, Glas. Mat. Ser. III 50(70) (2015), 17-24.

[16] K. Gyarmati, A. Sarkozy and C.L. Stewart, On shifted products which are powers, Mathematika 49 (2002), 227-230.

[17] K. Gyarmati and C. L. Stewart, On powers in shifted products, Glas. Mat. Ser. III 42 (62) (2007), 273-279.

[18] L. K. Hua and Y. Wang, Applications of number theory to numerical analysis, Translated from Chinese. Springer-Verlag, Berlin-New York; Kexue Chubanshe (Science Press), Beijing, 1981.

[19] F. Luca, On shifted products which are powers, Glas. Mat. Ser. III 40(60) (2005), 13-20. 
[20] F. Luca and L. Szalay, Fibonacci Diophantine Triples, Glas. Mat. Ser. III 43 (63) (2008), 253-264.

[21] F. Luca and L. Szalay, Lucas Diophantine Triples, Integers 9 (2009), 441-457.

[22] E. P. Miles, Jr., Generalized Fibonacci numbers and associated matrices, Amer. Math. Monthly 67 (1960), 745-752.

[23] M. D. Miller, Mathematical Notes: On Generalized Fibonacci Numbers, Amer. Math. Monthly 78 (1971), 1108-1109.

[24] D. A. Wolfram, Solving generalized Fibonacci recurrences, Fibonacci Quarterly 36 (1998), 129-145. 